\title{
Investimento Estrangeiro Direto no Brasil: Efeitos Diretos e Espaciais sobre o Crescimento Econômico
}

\section{Foreign Direct lnvestment in Brazil: Direct and Spatial Effects on Economic Growth}

\author{
Gilberto Joaquim Fraga* \\ José Luiz Parré** \\ Renilson Rodrigues da Silva***
}

\begin{abstract}
Resumo: A presente pesquisa objetiva analisar os efeitos diretos e indiretos (spillover regional) do investimento estrangeiro direto nos estados brasileiros sobre a taxa de crescimento econômico. Para tanto, se utiliza primeiramente o procedimento de análise espacial para se verificar a existência de correlação espacial entre os estados e, posteriormente, o procedimento econométrico de dados em painel com pesos espaciais. Os resultados encontrados sugerem que o investimento estrangeiro direto pode afetar o crescimento econômico de forma direta. Também é possível observar o efeito spillover regional positivo. No entanto, essa relação mostra-se sensível a mudanças na especificação.
\end{abstract}

Palavras-chave: Investimento estrangeiro direto. Spillover. Correlação especial.

\begin{abstract}
This paper aims to analyze the direct and indirect effects (regional spillover) of foreign direct investment in the Brazilian states on the rate of economic growth. First, was used the procedure of spatial analysis to verify the presence of spatial correlation among the states, and then, we apply econometrics of panel data with spatial weights. The results suggest that foreign direct investment can affect economic growth directly. Also is possible to observe a regional spillover effect positive. However, this relationship demonstrates sensitive to changes in the specification.
\end{abstract}

Keywords: Foreign direct investment. Spillover. Spatial correlation.

JEL Classification: F21; F23; R12.

\footnotetext{
* $\quad$ Doutor em Economia Aplicada pela Universidade de São Paulo (USP). Professor do Departamento de Economia da Universidade Estadual de Maringá (UEM). E-mail: gjfraga@uem.br

** Doutor em Economia Aplicada pela Universidade de São Paulo (USP). Professor titular do Departamento de Economia da Universidade Estadual de Maringá (UEM). E-mail: jlparre@uem.br *** Doutor em Economia Aplicada pela Universidade de São Paulo (USP). Professor do Departamento de Economia da Universidade Federal de São João Del-Rei (UFSJ). E-mail: renilsonsilva@ufsj. edu.br
} 


\section{Introdução}

Dentre os países em desenvolvimento, o Brasil vem se destacando como grande recebedor de investimento estrangeiro direto (IED), principalmente a partir da segunda metade dos anos 1990 (do século XX), com a consolidação da estabilidade econômica e a maior abertura comercial e financeira. Após um período de desempenho franco, segundo dados da UNCTAD, entre 1995 e 2005 o crescimento do estoque de IED ${ }^{1}$ no Brasil foi de $278,7 \%$. Esse crescimento foi $62,5 \%$ superior àquele observado no resto do mundo no mesmo período. Contudo, deve-se ressaltar que esse expressivo crescimento do IED no Brasil é geograficamente concentrado em alguns estados. A literatura internacional mostra que esse tipo de concentração espacial não é exclusividade do Brasil. Trata-se de um fenômeno observado em outros países de economias emergentes, como China (MADARIAGA; PONCET, 2007) e México (JORDAAN; RODRÍGUEZ OREGGIA, 2012), por exemplo. A teoria da Nova Geografia Econômica (NGE) busca explicar esse tipo de comportamento locacional das empresas estrangeiras nos países hospedeiros.

Dado o forte crescimento do fluxo global de IED, cresce a discussão sobre os potenciais benefícios econômicos e/ou de bem-estar para os países receptores, conforme pode ser visto em Alfaro et al. (2004, 2009), Carkovic e Levine (2005), Bloniggen et al. (2007), Chudnovsky e López (2007), Madariaga e Poncet (2007) e Jordaan e Rodríguez Oreggia (2012). O bom entendimento desses efeitos é de suma importância para a formulação de políticas públicas.

$\mathrm{Na}$ literatura brasileira existe uma lacuna sobre o entendimento da relação entre IED e crescimento econômico, principalmente quando se considera a questão espacial. No caso do Brasil isso se deve, em parte, à carência de um banco de dados sobre IED nos estados/regiões brasileiros. As pesquisas existentes estão concentradas nos determinantes da entrada do IED na economia brasileira (BORTOLUZZO; SAKURAI; BORTOLUZZO, 2013), e não nos potenciais efeitos sobre o crescimento da economia.

Diante desse cenário, o objetivo desse estudo é investigar se a atração de IED tem contribuído para o crescimento econômico dos estados brasileiros. Em particular, se o IED afeta o crescimento econômico dos estados brasileiros de forma direta e indireta através de spillovers espaciais entre os estados. Para tanto, utiliza-se como fundamentação teórica os pressupostos formulados na NGE. No que tange ao desenvolvimento empírico do trabalho, é utilizado o instrumental da econometria espacial.

Além desta introdução, o presente trabalho está estruturado como segue: na segunda seção apresentam-se alguns pressupostos teóricos da NGE; na terceira

1 IED em milhares de dólares corrente. 
seção é feita uma revisão de estudos empíricos sobre a relação entre IED e crescimento econômico; na quarta seção são apresentados os dados e a especificação empírica; na quinta seção são relatados os resultados. Por fim, na sexta seção são levantadas as considerações finais do trabalho.

\section{Investimento Estrangeiro Direto e a Nova Geografia Econômica}

As análises sobre os determinantes do IED são numerosas. Há trabalhos relevantes nesse campo de estudo, como os de Penrose (1956), Hymer (1960), Aliber (1970), entre muitos outros. Em estudos recentes, nota-se um grande foco nos modelos da NGE, com contribuições tanto teóricas, quanto empíricas. A principal característica dessas contribuições é a de considerar, incluindo os determinantes das escolhas de localização, os custos de transporte e a distância geográfica.

Nessa linha, Dunning e Stopford (1983) destacam que há pelo menos três tipos de IED. O primeiro é o market-seeking, cuja finalidade é atender um determinado mercado local ou regional. Esse tipo é também chamado de horizontal, uma vez que envolve replicação de unidades de produção no país hospedeiro. Os investidores market-seeking são atraídos para um país com mercado local grande e de crescimento acelerado. O segundo tipo de IED é o resource-seeking, o qual procura países onde há recursos naturais abundantes. O terceiro tipo de IED é o efficiency-seeking, em que a proximidade geográfica com o país de origem é mais importante, pois possibilita a minimização dos custos. Todas essas características sugerem que países que possuem grandes mercados, baixo custo da mão de obra, recursos naturais abundantes e que estão próximos dos principais mercados irão atrair grandes somas de IED (KINOSHITA; CAMPOS, 2003).

\subsection{A Nova Geografia Econômica}

A NGE é o estudo da localização da atividade econômica no espaço. Portanto, para NGE é o espaço que importa na configuração da atividade econômica. Em particular, é uma vertente da literatura iniciada por Krugman (1991), usando economias de aglomeração para ajudar a explicar o porquê das indústrias se agruparem dentro de determinados países e/ou regiões. Desde então, tem crescido substancialmente o número de teóricos nesse campo e, em menor grau, os trabalhos empíricos. O foco padrão de aglomeração da NGE é voltado para aqueles elementos ditos de segunda natureza, como potencial de mercado, densidade do mercado de trabalho e economias externas puras. Por outro lado, Silva e Bacha (2014) destacam que há também uma corrente teórica complementar, a qual con- 
sidera, como fatores geradores da aglomeração, os elementos de primeira nature$\mathrm{za}$, como recursos naturais e clima, por exemplo.

Muitas atividades econômicas são concentradas geograficamente. Todavia, nem todas as pessoas vivem numa única grande cidade, tampouco a produção de bens na economia está concentrada num único local. Há um conjunto de forças atuando em direções opostas: de um lado, as forças centrípetas, que tendem a promover a concentração geográfica e, de outro, as forças centrífugas, agindo no sentido contrário. Forças centrífugas são regidas por efeitos do tamanho de mercado, economias externas puras e mercado de trabalho. Forças centrípetas são regidas por imobilidade de fatores, renda da terra e deseconomias externas puras (KRUGMAN, 1998).

A NGE tem um conjunto de predecessores teóricos que remontam às teorias clássicas da localização, desde o tratamento dos custos de transportes aos processos de causação cumulativa e aglomerações industriais. No entanto, as teorias clássicas da localização não explicam o porquê das atividades econômicas se concentrarem e/ou se dispersarem no espaço endogenamente com o uso de modelos econômicos fundamentados na microeconomia do comportamento. $\mathrm{Na}$ NGE, essa deficiência foi sanada e seus elementos fundamentais são abordados de seguinte forma:

a) rendimentos crescentes em escala: as economias de escala favorecem a concentração regional, além de evidenciarem as importâncias dos retornos crescentes e dos efeitos de transbordamento do conhecimento, que são basicamente as externalidades positivas. $\mathrm{Na}$ ausência de economia de escala, os produtores não teriam incentivos para concentrar suas atividades. O pressuposto de economia de escala se materializa na existência de custos fixos que são necessários para a produção dos bens ou para a prestação de serviços por parte da empresa. Essa hipótese define a forma pela qual as empresas devem competir, constituindo o segundo elemento essencial, que é a concorrência imperfeita;

b) concorrência imperfeita: sob a hipótese de rendimentos crescentes em escala, os custos marginais são menores que os custos médios, e isso faz com que seja inviável um esquema de concorrência perfeita, no qual as empresas, ao fixar os preços, no nível dos custos marginais, estariam incorrendo em perdas. A grande maioria dos modelos da NGE é guiada por um esquema de concorrência à la Chamberlin, sendo muito recorrente na literatura o uso da estrutura do modelo de diferenciação e variedades de produto de Dixit e Stiglitz;

c) custos de transporte (acessibilidade aos mercados): os insumos utilizados pelas empresas como fatores de produção de seus bens finais e destinados aos consumidores têm de ser transportados, e isso implica custos para as 
empresas. A existência desse atrito no comércio de bens induz as empresas a se localizarem em regiões com bom acesso ao mercado consumidor e aos fornecedores de insumos. Daí uma das razões, apontada por Krugman (1998), da concentração da atividade econômica. Dessa maneira, para que as empresas possam minimizar seus custos de transportes, elas expandem seus mercados para outros locais. Teoricamente, entretanto, essas decisões dependem da suposição de que há rendimentos crescentes à escala na função de produção proposta. Na existência de rendimentos constantes ou decrescentes em escala, as pequenas empresas, ou filiais das grandes empresas, abastecem o mercado local. Porém, essa é uma situação que não se sustenta na presença de rendimentos crescentes, pois as empresas preferem concentrar a produção em alguns locais. De modo geral, os benefícios da proximidade geográfica e os custos da distância podem ser influenciados positiva ou negativamente;

d) localização da demanda: o poder de compra de uma dada localidade influencia a localização das empresas e da mobilidade dos trabalhadores na indústria. Basicamente, se propõem dois mecanismos na mobilidade da demanda: a) quando há uma fração dos trabalhadores móveis que se deslocam para áreas onde existem mais empresas e variedades de produtos - os trabalhadores procuram por melhores salários e melhores preços de produtos. Esse fenômeno produz efeito forward linkages (ligações para frente), pois incentiva os trabalhadores a se deslocarem para os centros produtores de bens de consumo, que, por sua vez, induz o aumento da demanda nesse local (KRUGMAN, 1991); e b) quando as firmas produtoras de bens finais demandam bens intermediários - com isso, as produtoras de bens finais e intermediários se instalam no mesmo local. Se há maior demanda num determinado local, as empresas se deslocam para atender esse mercado, gerando os efeitos para trás. Com maior número de empresas nessa localidade, aumenta a demanda por mão de obra, tornando os salários reais mais elevados. Nessas condições, os trabalhadores migram em busca de maiores salários (efeitos para frente), aumentando ainda mais a demanda desse mercado. A maior demanda das empresas produtoras de bens finais atrai também as firmas produtoras de bens intermediários (efeitos para trás), que faz aumentar a demanda por trabalhador (efeitos para frente) caracterizando o fluxo circular, chamado de efeito do mercado interno por Krugman (1991).

Como mostram Blonigen et al. (2007) e Blanco (2012), a interação espacial se mostra presente nos fatores determinantes da localização do IED. Dessa forma, dada as características econômicas e geográficas dos países, que afetam a locali- 
zação das empresas com capital estrangeiro, surge a necessidade de se conhecer quais são os efeitos diretos e indiretos do IED sobre o crescimento econômico.

\section{Investimento Estrangeiro Direto e Crescimento Econômico: Literatura Em- pírica Relacionada}

Na literatura internacional encontram-se os trabalhos de Alfaro et al. (2004), Javorcik (2004) e Durham (2004), os quais mostram, empiricamente, que o IED pode ter efeito positivo sobre a taxa de crescimento econômico das nações. Quando se considera os efeitos do IED dentro dos países, a literatura indica que nem sempre o IED gera efeitos diretos sobre a taxa de crescimento econômico (CHUDNOVSKY; LÓPEZ, 2007; CRESPO; FONTOURA, 2007). Tais efeitos positivos podem não se materializar em certos países, ou seja, pode depender de outras políticas realizadas previamente pelos países receptores. Ressalta-se que, em alguns países, o IED é geograficamente concentrado. No caso da América Latina, Blanco (2012) apresenta e discute a interdependência espacial do IED. Mesmo se observando os efeitos médios positivos sobre o crescimento nacional, em nível regional/estadual, esses efeitos podem não acontecer, como mostra Sjöholm (1999).

Para analisar os efeitos do IED sobre o crescimento da produtividade em nível regional e nacional na Indonésia, Sjöholm (1999) realiza estimativas para os anos 1980 e 1991. O autor constata que, embora existam spillovers intraindustriais em nível nacional, o mesmo não acontece no âmbito regional.

Javorcik (2004) analisa o efeito do IED sobre a produtividade em nível de firmas da Lituânia. Os resultados mostram a existência de spillovers positivos e o aumento de produtividade resultante do contato das firmas estrangeiras (IED) com as firmas domésticas. No entanto, a localização geográfica não é levada em consideração.

Em um estudo para países em desenvolvimento, Bengoa e Sánchez Robles (2003) analisam a relação entre IED e crescimento econômico a partir de dados de 18 países da América Latina, entre 1970 e 1999, através do procedimento de dados em painel. A pesquisa contempla, também, o papel da liberdade econômica nos respectivos países. Os resultados sugerem que existe uma correlação positiva entre IED e crescimento econômico. Ademais, contata-se que a liberdade econômica é um determinante positivo para a entrada de IED. De acordo com os autores, a liberdade econômica afeta o crescimento econômico de forma positiva, pois aumenta a capacidade dos países de atrair IED.

Alfaro et al. (2004) investigam a relação entre IED, mercado financeiro e crescimento econômico no período entre 1975 e 1995. Os autores estimam essa relação por meio do método de variáveis instrumentais. Usam dois grupos de países, ambos contendo tanto países desenvolvidos, quanto em desenvolvimento. 
De acordo com os resultados, a entrada de IED, por si só, não afeta a taxa de crescimento econômico. Entretanto, os autores verificam que países com mercado financeiro bem desenvolvido se beneficiam significantemente da entrada de IED. Nessa mesma perspectiva, Durham (2004) estima o efeito do IED e do investimento em carteira sobre o crescimento econômico. Para tanto, usa dados de 80 países, no período entre 1979 e 1998. Os resultados mostram que a correlação positiva entre IED e crescimento econômico é condicionada à capacidade absortiva dos países receptores. Entretanto, essa correlação depende de um bom desenvolvimento financeiro e de boas instituições. Esses resultados estão em linha com aqueles apresentados por Borenstein et al. (1998). Em um trabalho seguinte, Alfaro, Kalemli-Ozcan e Sayek (2009) verificam o efeito do IED sobre a produtividade dos países. Concluem que países com mercado financeiro bem desenvolvido podem obter ganhos de produtividade com a entrada de IED.

Madariaga e Poncet (2007) analisam o impacto do IED sobre o crescimento regional na China e seus spillovers espaciais. O estudo aborda o período de 1990 a 2002, considerando 180 cidades que cobrem quase todo o território (exceto Qinghai e Tibet) da China. As autoras usam uma especificação empírica seguindo os modelos tradicionais de crescimento e adicionam uma variável para captar os efeitos espaciais. Os resultados encontrados apontam dependência espacial e sugerem a existência de efeitos diretos e positivos do IED sobre o crescimento das regiões (cidades) chinesas. Ao fazerem estimativas via painéis dinâmicos, as autoras constatam, também, spillovers espaciais positivos para regiões vizinhas.

Nessa mesma perspectiva, Bode, Nunnenkamp e Waldkirch (2012) analisam os efeitos espaciais do IED em 47 estados norte-americanos (com exceção de Alasca, Havaí, Delaware e Washington D.C.) no período de 1977 a 2003. Os autores aplicam o método dos momentos generalizados com a presença de lag espacial. Os resultados apontam a existência de externalidade regional positiva com a presença de IED em determinados estados dos Estados Unidos. Porém, observam externalidade negativa para as firmas domésticas - efeito crowding out ${ }^{2}$ de mercado.

Em uma análise para países em desenvolvimento, Jordaan e Rodríguez Oreggia (2012) mensuram os efeitos do estoque de IED sobre o crescimento regional no México. Os resultados obtidos a partir de três subperíodos quinquenais, entre 1988 a 2004, mostram que, embora o efeito médio nacional seja positivo, existem efeitos espaciais negativos do IED entre as regiões mexicanas.

2 A discussão desse ponto foge ao escopo do presente artigo. Agosin e Mayer (2000) e Bode, Nunnenkamp e Waldkirch (2009) discutem o efeito crowding out.

FRAGA, G. J.; PARRÉ, J. L.; SILVA, R. R. Investimento Estrangeiro Direto no Brasil: Efeitos... 


\section{Dados e Estratégia Empírica}

\subsection{Dados}

Os dados utilizados nesta pesquisa referem-se aos 26 estados brasileiros e o Distrito Federal nos anos de 1995, 2000 e 2005, contemplando um total de 81 observações disponíveis. A principal fonte de dados utilizada foi o Censo de Capitais Estrangeiros no País (CCEP), ${ }^{3}$ realizado pelo Banco Central do Brasil (Bacen). Foram utilizados os censos referentes a três datas-base: 1995, 2000 e 2005. A partir desses censos, foram obtidas as observações sobre IED. A variável investimento (operações de crédito de longo prazo) foi obtida a partir do Bacen via o software PASCS10 do Sistema de Operações, Registro e Controle do Banco Central (Sisbacen). As demais variáveis foram obtidas, ou construídas, a partir dos dados obtidos junto ao portal de dados do Instituto de Pesquisa Econômica e Aplicada (Ipeadata). As descrições das variáveis são:

a) PIB per capita (y): é representado pelo PIB per capita, em reais de 2000, e deflacionado pelo deflator implícito do PIB. A partir dessa variável é gerada a taxa de crescimento do PIB per capita dos estados brasileiros, calculada pelos autores da pesquisa a partir dos dados do Ipeadata;

b) IED em relação ao PIB (IED/PIB): é representado pela razão IED do estado $i$ em relação ao PIB do respectivo estado (IED/PIB). Os valores foram deflacionados pelo índice geral de preços de mercado. Os cálculos foram feitos pelos autores a partir dos dados do Bacen (IED) e do Ipeadata (PIB);

c) matriz de pesos espaciais $(W)$ : a matriz utilizada neste trabalho é binária do tipo rainha. A matriz de pesos espaciais serve para captar o arranjo espacial das interações resultantes do fenômeno estudado. Regiões mais conectadas entre si apresentam maior interação. A matriz de ponderação espacial na configuração rainha foi utilizada por apresentar o maior valor para o I de Moran;

d) investimento (INVEST): é definido como razão entre investimento e PIB dos estados. Investimento é representado pela soma das operações de crédito dos bancos nos estados e o investimento público em capital realizado pelos estados. As séries de dados foram obtidas por meio do software PASCS10 do Sisbacen e deflacionadas pelo índice geral de preços de mercado (IGPM);

e) população (POP): é representada pelo tamanho da população dos estados;

3 Para mais detalhes sobre o CCEP, acessar: <http://www.bcb.gov.br/rex/censoce/port/censo.asp>. 
f) capital humano ${ }^{4}(\mathrm{H})$ : é definido como a escolaridade média da população com 25 anos de idade ou mais;

g) infraestrutura dos estados (infraes-km): como proxy para infraestrutura é utilizada a malha rodoviária asfaltada, em quilômetros (mil km).

\subsection{Procedimento de Efeitos Espaciais}

De acordo com Anselin (1999, p. 1), os estudos que utilizam econometria espacial devem levar em consideração dois efeitos espaciais na sua estimação: dependência espacial, ou autocorrelação espacial, e heterogeneidade espacial.

A dependência espacial, ${ }^{5}$ segundo Almeida (2012), ocorre pela interação dos agentes no espaço, ou seja, o valor de uma variável de interesse numa certa região i depende do valor dessa variável nas regiões vizinhas j. A inserção da localização no estudo é importante, pois quando este não é inserido, os resultados proporcionados pela econometria convencional podem se tornar, de certo modo, inconsistentes. As técnicas espaciais incorporam na modelagem o padrão da interação socioeconômica entre os agentes do sistema, bem como as características da estrutura desse sistema no espaço.

Já a heterogeneidade espacial ocorre devido ao fato de fenômenos espaciais não apresentarem estabilidade estrutural (como coeficientes variáveis, variância não constante ou, ainda, formas funcionais diferentes para determinados subconjuntos de dados). Consequentemente, dificulta-se o ajustamento de um mesmo modelo teórico para todo e qualquer conjunto de dados, acarretando perda de eficiência ou, até mesmo, estimativas viesadas (ALMEIDA, 2012).

A autocorrelação é calculada pela estatística I de Moran, a qual fornece indicação do grau de associação linear entre os vetores de valores observados no tempo e a média ponderada dos valores da vizinhança. A fórmula dessa estatística é expressa como

$$
I=\frac{n}{\sum \sum w_{i j}} \frac{\sum \sum w_{i j}\left(y_{i}-\bar{y}\right)\left(y_{j}-\bar{y}\right)}{\sum\left(y_{i}-\bar{y}\right)^{2}}
$$

$4 \quad$ Não há disponibilidade da variável capital humano no ano de 2000 porque não houve pesquisa nacional por amostra de domicílios. Portanto, foi feita uma interpolação para esse ano.

5 Refere-se à associação linear entre as variáveis que surge devido à interação espacial. Esta devese à movimentação de bens, pessoas e/ou informações através do espaço de tal forma que as circunstâncias de um lugar podem afetar as condições em outros lugares que interagem entre si.

FRAGA, G. J.; PARRÉ, J. L.; SILVA, R. R. Investimento Estrangeiro Direto no Brasil: Efeitos... 
sendo que $n$ é o número de unidades espaciais, $y_{i}$ é a variável de interesse e Wij é o peso espacial para o par de unidades espaciais $i$ e j, medindo o grau de interação entre elas.

O coeficiente I de Moran tem um valor esperado (média teórica) de -[1/ (n-1)], isto é, o valor que seria obtido se não houvesse padrão espacial nos dados. O valor calculado de I deveria ser igual ao seu valor esperado, dentro dos limites da significância estatística, se $y_{i}$ é independente dos valores nas regiões vizinhas. Valores de I que excedem -[1/ (n-1)] indicam autocorrelação espacial positiva. Valores de I abaixo do valor esperado sinalizam uma autocorrelação negativa. Uma indicação de autocorrelação espacial positiva revela que há uma similaridade entre os valores do atributo estudado e da localização espacial do atributo. Já uma indicação de autocorrelação espacial negativa revela, por sua vez, que há uma dissimilaridade entre os valores do atributo estudado e os da localização espacial do atributo.

Apresenta-se, na Figura 1, o diagrama de dispersão da estatística I de Moran com os tipos de agrupamentos de associação linear espacial.

Figura 1 - Diagrama da dispersão da estatística I de Moran

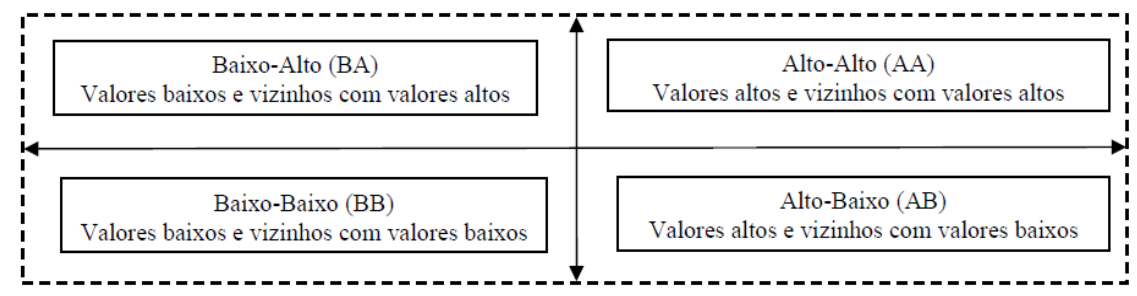

Fonte: Capucho e Parré (2010).

Para finalizar a apresentação, é necessário destacar a importância da matriz de pesos (W) na análise espacial, pois todos os passos subsequentes (ou resultados) dependerão dessa matriz. A matriz de pesos é a forma de expressar a estrutura espacial dos dados. Há na literatura um grande número de matrizes de pesos espaciais que podem ser binárias, de $K$ vizinhos mais próximos, ou utilizar alguma medida de distância entre as unidades espaciais vizinhas. Diferentes configurações de matrizes foram testadas para este estudo, e a escolhida foi a matriz de pesos espaciais (W) binária do tipo rainha, que apresentou a maior autocorrelação espacial do fenômeno em estudo (maior valor para o I de Moran). ${ }^{6}$

6 A convenção rainha faz alusão ao movimento de peças num tabuleiro de xadrez, indicando, assim, de que forma levar em consideração as fronteiras geográficas. No caso da rainha, além das fronteiras entre as unidades espaciais com extensão diferente de zero, essa convenção considera também os vértices (nós). 


\subsection{Especificação Empírica}

Para implementar a estratégia empírica será utilizada uma especificação similar a de outros trabalhos dessa área (JORDAAN; RODRÍGUEZ OREGGIA, 2012). O efeito direto do IED sobre a taxa de crescimento econômico e os spillovers podem ser apresentados por meio das seguintes equações:

efeito direto:

$$
y_{i, t}=\alpha+\beta_{1} y_{i, t-T}+\beta_{k} X_{i, t}+\beta_{4} I E D_{i, t}+\varepsilon_{i t}
$$

efeito espacial (spillovers):

$$
y_{i, t}=\alpha+\beta_{1} y_{i, t-T}+\beta_{2} X_{i, t}+\beta_{3} I E D_{i, t}+\beta_{4} W \cdot I E D_{i, t}+\varepsilon_{i t}
$$

em que $y$ é a taxa de crescimento do estado i no ano t; IED é o do respectivo estado; $X$ representa um conjunto das demais variáveis independentes (controle), que é constituído por variáveis utilizadas pela literatura pertinente, incluindo população, capital humano, investimento doméstico e nível inicial de renda; $W^{\top}$ é a matriz de pesos espaciais; e $\varepsilon_{i t}$ o termo de erro.

Deve-se ressaltar que a taxa de crescimento é constituída de variáveis que são medidas em valores médios ${ }^{8}$ de cinco anos, sendo que o período se estende de 1995 a 2005. Portanto, há duas observações por estado (1995-2000 e 2001-2005). Assim, relaciona-se o IED de 1995 com a taxa média de crescimento do período 1995-1999 e o IED de 2000 com a taxa média de crescimento de 2000-2005.

\section{Estimativas e Testes}

\subsection{Efeito Direto}

Os resultados das estimativas por meio dos vários procedimentos do modelo empírico apresentado na equação 2 estão na Tabela 1. A primeira coluna (regressão 1) apresenta resultados via procedimento pooled (OLS). O coeficiente do PIB inicial tem o sinal esperado e foi estatisticamente significante - sinalizando a presença do processo de convergência. No entanto, a principal variável de interesse,

$7 \quad$ Para detalhes da matriz de pesos espaciais, ver Anselin (1999).

8 O uso de dados formado por médias de cinco anos visa suavizar os efeitos dos ciclos de negócios (CARKOVIC; LEVINE, 2005). O período encerra em 2005 devido à disponibilidade dos dados de forma homogênea.

FRAGA, G. J.; PARRÉ, J. L.; SILVA, R. R. Investimento Estrangeiro Direto no Brasil: Efeitos... 
a variação no IED, embora tenha apresentado coeficiente com sinal positivo, não é estatisticamente significante.

Tabela 1 - Estimativas pooled e painel

(variável dependente: taxa de crescimento do PIB per capita)

\begin{tabular}{|c|c|c|c|c|c|}
\hline \multirow[b]{2}{*}{ Variável } & \multirow{2}{*}{ Pooled } & \multicolumn{4}{|c|}{ Painel } \\
\hline & & (2) & (3) & (4) & (5) \\
\hline $\mathrm{PIB}_{(\mathrm{t}-1)}$ & $-0,0470^{* *}$ & $-0,0348^{* * *}$ & $-0,0470^{* *}$ & $-0,0243$ & $-0,0467^{* *}$ \\
\hline & $(0,0207)$ & $(0,00945)$ & $(0,0205)$ & $(0,0212)$ & $(0,0205)$ \\
\hline INVEST & $0,00270^{*}$ & 0,00151 & 0,00270 & 0,00613 & 0,00265 \\
\hline & $(0,00141)$ & $(0,00232)$ & $(0,00481)$ & $(0,00465)$ & $(0,00461)$ \\
\hline $\mathrm{H}$ & $0,0219^{* *}$ & $0,0181^{* * *}$ & $0,0219^{* * *}$ & 0,00711 & $0,0229^{* * *}$ \\
\hline & $(0,00892)$ & $(0,00435)$ & $(0,00699)$ & $(0,00689)$ & $(0,00635)$ \\
\hline D.IED ${ }^{a}$ & 0,00755 & $-0,00153$ & $0,00755^{*}$ & $0,0266^{* * *}$ & \\
\hline IED & & & & & $\begin{array}{r}-0,0289 \\
(0.0200)\end{array}$ \\
\hline $\mathrm{POP}^{\mathrm{b}}$ & $\begin{array}{r}-0,0157^{*} \\
(0,00888)\end{array}$ & $\begin{array}{r}-0,0143^{* * *} \\
(0,00463)\end{array}$ & $\begin{array}{r}-0,0157 * * * \\
(0,00536)\end{array}$ & $\begin{array}{r}-0,0195^{* * *} \\
(0,00360)\end{array}$ & $\begin{array}{r}-0,0139^{* *} \\
(0,00657)\end{array}$ \\
\hline Infraes-km ${ }^{b}$ & $\begin{array}{r}0,0187^{*} \\
(0,00972)\end{array}$ & $\begin{array}{r}0,0165^{* * *} \\
(0,00511)\end{array}$ & $\begin{array}{r}0,0187^{* * *} \\
(0,00161)\end{array}$ & $\begin{array}{r}0,0160^{* * *} \\
(0,00124)\end{array}$ & $\begin{array}{r}0,0186^{* * *} \\
(0,00118)\end{array}$ \\
\hline $\begin{array}{l}\text { Dummy2000 } \\
-2005\end{array}$ & & & & $\begin{array}{r}0,0320^{* * *} \\
(0,00343)\end{array}$ & \\
\hline Constante & $\begin{array}{r}0,0448 \\
(0,0841)\end{array}$ & $\begin{array}{r}0,0471 \\
(0,0484)\end{array}$ & $\begin{array}{r}0,0448 \\
(0,0690)\end{array}$ & $\begin{array}{r}0,157^{* * *} \\
(0,0304)\end{array}$ & $\begin{array}{r}0,0158 \\
(0,0919)\end{array}$ \\
\hline $\mathrm{R}^{2}$ & 0,268 & & 0,268 & 0,439 & 0,272 \\
\hline Wald & - & $\mathrm{P}>\left(\mathrm{chi}^{2}\right)=0,000$ & $\mathrm{P}>\left(\mathrm{chi}^{2}\right)=0,016$ & $\mathrm{P}>\left(\mathrm{chi}^{2}\right)=0,00$ & $\mathrm{P}>\left(\mathrm{chi}^{2}\right)=0,00$ \\
\hline
\end{tabular}

Fonte: Elaboração própria a partir dos dados da pesquisa.

Notas: Robust standard errors in parentheses: *** p $<0.01, * * \mathrm{p}<0.05 \mathrm{e} * \mathrm{p}<0.1$; FGLS: coluna 2 controla heteroscedasticidade entre os painéis (estados); e colunas 3 a 5 controlam heteroscedasticidade e correlação dos erros entre os painéis (estados). ${ }^{a}$ ) D.IED: é diferença $\left(\right.$ ied $_{t}$ ied $\left._{t-1}\right) ;{ }^{b}$ ) variáveis em $L$.

Para constatar a possível presença de heteroscedasticidade nos grupos de painéis foi realizado o teste modificado de Wald. ${ }^{9}$ De acordo com o resultado, ao nível de significância de 1\%, não é possível rejeitar a hipótese de heteroscedasticidade para o grupo de painéis. Portanto, as estimativas seguintes devem levar essa questão em consideração.

As estimativas apresentadas nas regressões de 2 a 5 foram realizadas via procedimento de dados em painel. A regressão 2 mostra os resultados via procedimento de mínimos quadrados generalizados factíveis (FGLS), ${ }^{10}$ como sugere Jordaan e Rodríguez Oreggia (2012). A estimativa da variável PIB inicial confirma o resultado encontrado na primeira regressão. Entretanto, o IED não se apresenta estatisticamente significante para o crescimento econômico. Já a variável investimento doméstico deixa de ser estatisticamente significante apesar de apresentar o

$9 \quad$ Em específico, o teste consiste em verificar se $\mathrm{E}\left(u_{i t} u_{\mathrm{j} j}\right) \neq \mathrm{I} \sigma^{2}$ para $i \neq j$, em que $I$ é a matriz identidade. Objetiva-se, portanto, verificar se as variâncias dos grupos são homocedásticas.

10 Para mais detalhes sobre a aplicação do método FGLS, ver Cameron e Trivedi (2010). 
sinal esperado. As variáveis capital humano $(\mathrm{H})$ e infraestrutura $(\mathrm{km})$ são significativas e mostram que o investimento em ambas, em média, pode contribuir para aumentar a taxa de crescimento econômico dos estados. O coeficiente negativo e estatisticamente significante da variável população (POP) dos estados sugere que não há presença de economias de aglomeração desta fonte.

As estimativas 3 e 4 apresentam resultados através do procedimento que controla a possibilidade de os painéis serem heteroscedásticos e relacionados através dos erros. Os resultados apresentam um fato importante: a variável que representa a mudança no IED é estatisticamente significante e apresenta o sinal esperado (regressões 3 a 4). Isso implica que, na média, aumento no estoque de IED como proporção do PIB pode contribuir para aumentos na taxa de crescimento econômico. As demais variáveis mostraram-se estáveis em relação à estimativa anterior (regressão 2). A regressão 4 é uma replicação da estimativa 3 com a inclusão de uma dummy temporal, para captar choques específicos no tempo. A principal observação é que houve uma melhora na estatística $R^{2}$. No entanto, os coeficientes das variáveis PIB inicial e capital humano não permaneceram significantes. A estimativa 5 apresenta os resultados com a variável IED em nível. O objetivo desta regressão é observar como a taxa média de crescimento em determinando quinquênio pode ser afetada pelo nível do IED em relação ao PIB no início do quinquênio. Pode-se constatar que essa variável não se apresenta como significante e não apresenta o sinal esperado. Por outro lado, as variáveis PIB inicial, capital humano, população e infraestrutura mostraram-se estáveis em termos de sinal e significância estatística.

Para verificar se o problema de endogeneidade afeta os resultados via causalidade reversa entre crescimento e IED, o teste de Hausman $(1978)^{11}$ foi implementado. Para tanto, o teste é realizado em duas etapas. Primeiramente, estima-se a regressão da variável IED contra as demais variáveis de controle e obtém-se o resíduo estimado. Então, adiciona-se o resíduo dessa regressão ao modelo de regressão original. Se o resíduo na segunda etapa da regressão se apresentar como estatisticamente significante, a endogeneidade será um problema potencial (JORDAAN; RODRÍGUEZ OREGGIA, 2012). O resultado desse exercício mostrou que os resíduos da primeira etapa da regressão, tendo a variável IED como dependente, não apresentou como sendo estatisticamente significante ao nível convencional de $10 \%$. Portanto, as estimativas apresentadas não são influenciadas por problemas de endogeneidade na relação entre crescimento do produto dos estados e o IED.

Considerando a existência de uma considerável concentração do IED, sobretudo nos estados da região sudeste, e o sinal negativo encontrado para a variável de aglomeração (POP), torna-se relevante verificar a possível existência de externalidade (spillover) espacial do IED.

11 Apresentação detalhada do teste pode ser encontrada no trabalho de Adkins e Hill (2011).

FRAGA, G. J.; PARRÉ, J. L.; SILVA, R. R. Investimento Estrangeiro Direto no Brasil: Efeitos... 


\subsection{Evidências sobre Dependência Espacial}

A partir da perspectiva da geografia econômica, a taxa de crescimento do PIB per capita de determinado estado pode ser afetada pelos estados vizinhos. Dessa forma, o diagnóstico da dependência espacial é importante para conclusões consistentes no que se refere à relação entre IED e crescimento econômico.

Em relação ao spillover espacial, Madariaga e Poncet (2007, p. 842) destacam que:

The closer a local firm is located to an FDI firm, the more likely and the more frequently labour move between these two firms. This spatial link may also be important for vertical spillovers between firms and their local suppliers, which often are located close to one another. It is furthermore well recognized the geographic proximity facilitates flows of knowledge.

Para se obter a indicação sobre o efeito espacial do IED, verificou-se a estatística I de Moran, por meio da qual se teve a confirmação da correlação espacial. ${ }^{12}$ Os diagramas de dispersão de Moran (ver Figuras 2, 3 e 4) indicam os resultados da correlação espacial do IED nos estados brasileiros. Conforme Pinto e Almeida (2012), o sinal positivo indica que altos (baixos) valores da variável em consideração tendem a estar circundados por altos (baixos) valores dessa variável em estados vizinhos.

12 Ver apresentação detalhada de Carvalho e Albuquerque (2010). 
Figura 2 - Diagrama de Moran IED/PIB para 1995

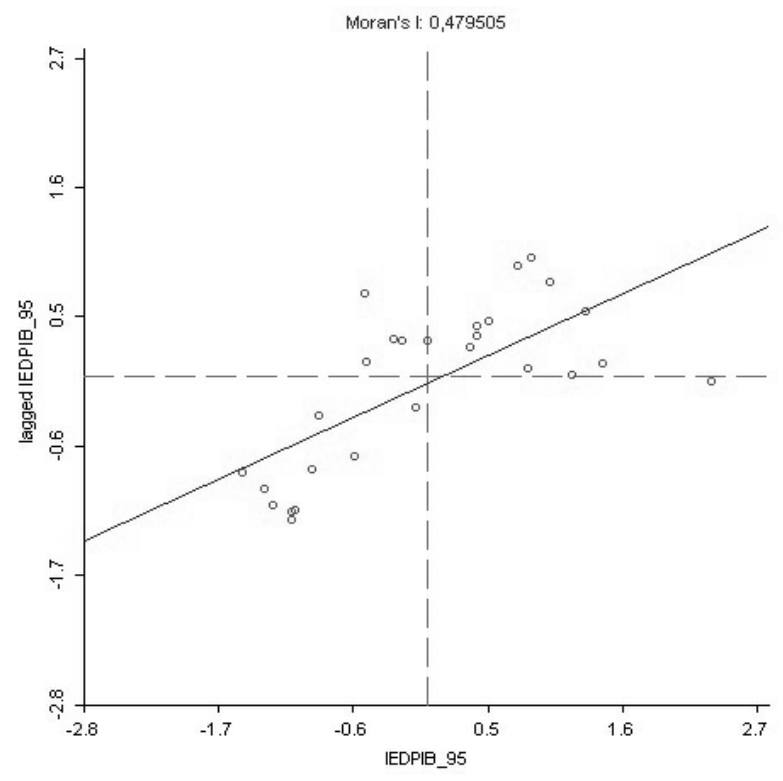

Fonte: Elaboração própria a partir dos dados da pesquisa.

Figura 3 - Diagrama de Moran IED/PIB para

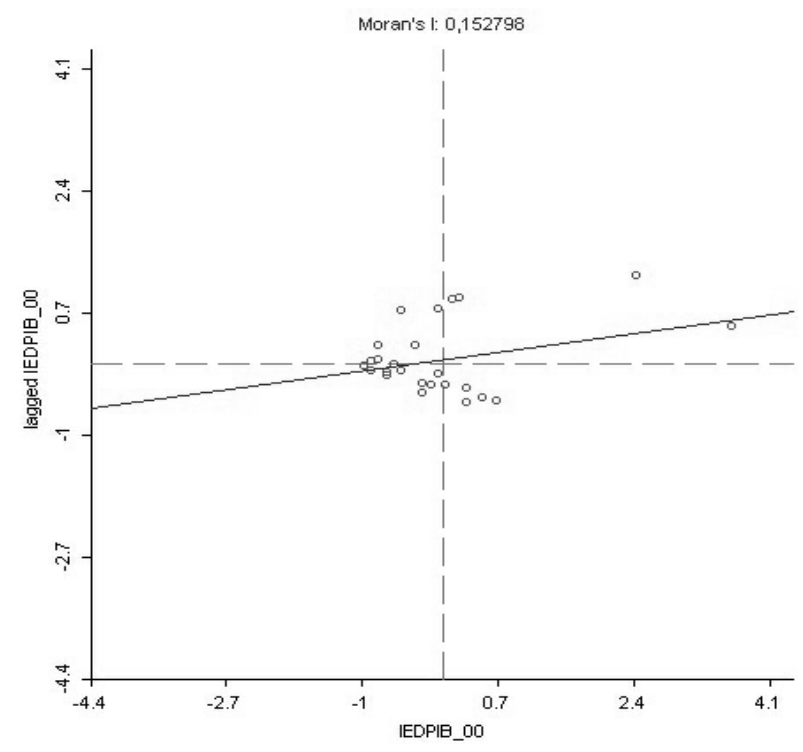

Fonte: Elaboração própria a partir dos dados da pesquisa. 
Figura 4 - Diagrama de Moran IED/PIB para 2005

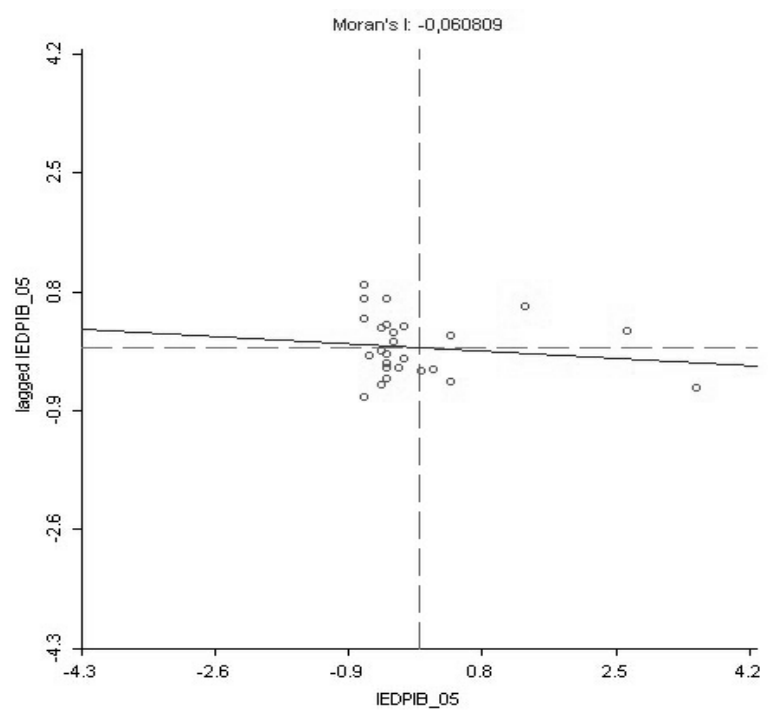

Fonte: Elaboração própria a partir dos dados da pesquisa.

Primeiramente, analisando os anos de 1995 e 2000, os resultados indicam que é possível rejeitar a hipótese nula de aleatoriedade espacial ao nível de significância de 1\% e 10\%, respectivamente. O sinal positivo sugere a existência de autocorrelação espacial positiva do IED para os estados brasileiros. A Tabela 2 reporta a estatística I de Moran para os períodos em análise.

Tabela 2 - Estatística I de Moran para o IED dos estados brasileiros

\begin{tabular}{cccc}
\hline Ano & $I$ de Moran & $p$-valor & Significante \\
\hline 1995 & 0,4795 & 0,001 & $1 \%$ \\
2000 & 0,153 & 0,060 & $10 \%$ \\
2005 & $-0,061$ & 0,480 & n.s. \\
\hline
\end{tabular}

Fonte: Elaboração própria a partir de dados da pesquisa.

Para o ano de 2005, a estatística I de Moran, apresentada na Tabela 2, e o diagrama de dispersão indicam que não é possível rejeitar a hipótese nula de aleatoriedade espacial. Portanto, os resultados sugerem que, no período de 1995 a 2005, o efeito spillover positivo do IED vem diminuindo. Além disso, deixou de ser estatisticamente significante no último período da amostra, conforme a Figura 3. O potencial efeito do IED sobre a taxa de crescimento econômico dos estados vem se tornando independente ao longo do período da análise. 
A taxa de crescimento é constituída de variáveis que são medidas em valores médios de cinco anos, compreendendo o período de 1995 a 2005. Assim, estima-se a média da taxa de crescimento desse período contra o valor base do IED com peso espacial (W). As observações do IED para 2005 não são consideradas, contornando, dessa forma, a questão da não significância estatística do I de Moran no ano de 2005. A Tabela 3 apresenta os resultados das estimativas levando em consideração o lag espacial do IED nos estados do Brasil, conforme especificação empírica da equação 3.

Tabela 3 - Estimativas com defasagem espacial do IED (variável dependente: taxa de crescimento do PIB per capita)

\begin{tabular}{|c|c|c|}
\hline \multirow[b]{2}{*}{ Variável } & \multicolumn{2}{|c|}{ Painel } \\
\hline & $(1)$ & (2) \\
\hline \multirow[t]{2}{*}{$\mathrm{PIB}_{(\mathrm{t}-1)}$} & $-0,0293 * * *$ & $-0,0443^{*}$ \\
\hline & $(0,0105)$ & $(0,0232)$ \\
\hline \multirow[t]{2}{*}{ INVEST } & 0,00201 & 0,00310 \\
\hline & $(0,00227)$ & $(0,00491)$ \\
\hline \multirow[t]{2}{*}{$\mathrm{H}$} & $0,0112 * *$ & $0,0186 * *$ \\
\hline & $(0,00502)$ & $(0,00749)$ \\
\hline \multirow[t]{2}{*}{ D.IED ${ }^{a}$} & 0,00480 & $0,0127 * * *$ \\
\hline & $(0,0171)$ & $(0,00452)$ \\
\hline \multirow[t]{2}{*}{$\mathrm{POP}^{\mathrm{b}}$} & $-0,0201 * * *$ & $-0,0161 * * *$ \\
\hline & $(0,00505)$ & $(0,00371)$ \\
\hline \multirow[t]{2}{*}{ Infraes. $(\mathrm{km})^{\mathrm{b}}$} & $0,0193 * * *$ & $0,0171 * * *$ \\
\hline & $(0,00514)$ & $(0,00119)$ \\
\hline \multirow[t]{2}{*}{ W_IED (efeito espacial) } & $0,143 * * *$ & $0,103^{*}$ \\
\hline & $(0,0458)$ & $(0,0596)$ \\
\hline \multirow[t]{2}{*}{ Constante } & 0,131 ** & $0,0713^{*}$ \\
\hline & $(0,0547)$ & $(0,0420)$ \\
\hline $\mathrm{R}^{2}$ & - & 0,290 \\
\hline Wald $\left(\mathrm{chi}^{2}\right)$ & $P>($ chi2 $)=0,000$ & $P>($ chi2 $)=0,0126$ \\
\hline
\end{tabular}

Fonte: elaboração própria a partir dos dados da pesquisa.

Notas: Robust standard errors in parentheses: *** $\mathrm{p}<0.01,{ }^{* *} \mathrm{p}<0.05 \mathrm{e}^{*} \mathrm{p}<0.1$; FGLS: regressão 1 controla heteroscedasticidade entre os painéis (estados); e regressão 2 controla heteroscedasticidade e correlação dos erros entre os painéis (estados). ${ }^{a}$ ) D.IED: é diferença (ied-ied $\left.{ }_{t-1}\right) ;{ }^{b}$ ) variáveis em $L$. 
As regressões 1 e 2 foram realizadas por meio de procedimento de dados organizados em painel. Com exceção da variável que representa investimento doméstico (INVEST), as demais variáveis são estatisticamente significantes e mostram estabilidade em relação aos resultados apresentados na Tabela 2.

O que se observou como novidade na estimativa 1 é que, apesar da variável IED não apresentar efeito direto (estatística significante), ela sugere a existência de spillover. Ou seja, um estado circundado de vizinhos que recebem IED pode se beneficiar de externalidades positivas destes. A estimativa 2 sugere que é possível observar tanto efeito direto, quanto indireto (espacial) do IED nos estados brasileiros, sendo o efeito indireto com maior capacidade de estimular a taxa de crescimento do PIB per capita dos estados. A magnitude do parâmetro do IED com defasagem espacial (W.IED) é 0,103, enquanto o parâmetro do efeito direto é de 0,0127. Esse efeito indireto maior está em linha com os resultados apresentados por Madariga e Poncet (2007).

Os resultados apresentados nesta seção mostraram que o efeito do IED sobre o crescimento econômico não apresentou estabilidade absoluta diante de mudanças nas especificações, ou nos procedimentos econométricos. No entanto, os resultados estão consistentes com a literatura empírica relacionada, a qual leva em consideração as questões da concentração regional do IED, como, por exemplo, no estudo de Jordaan e Rodríguez Oreggia (2012).

\section{Considerações Finais}

Na presente pesquisa se propôs analisar, empiricamente, os efeitos do IED nos estados brasileiros sobre o crescimento econômico. Além disso, se buscou considerar não somente os efeitos médios diretos, mas, também, analisar os spillovers, buscando verificar a importância do IED em determinado estado sobre os estados vizinhos.

Os resultados estão em linha com a literatura e sugerem que existe uma relação positiva entre a razão estoque de IED/PIB nos estados e o crescimento do PIB per capita. Foi possível observar os efeitos diretos do IED sobre o crescimento, seja por meio de procedimento de dados em painel padrão, seja pelo método de estimação espacial. Constatou-se, também, que existe correlação espacial do IED. No entanto, esse efeito decaiu ao longo do período analisado. Através das estimativas utilizando a matriz de pesos espaciais, os resultados mostraram que existem externalidades (spillovers) positivas do IED entre os estados brasileiros.

Quando se realizaram as estimativas aplicando painel padrão, os efeitos do IED se mostraram sensíveis às mudanças na especificação. De outro lado, quando se considerou o procedimento com abordagem espacial, os efeitos positivos do IED estiveram presentes nas duas especificações. Quanto às demais variáveis, 
se observou que o PIB inicial apresentou sinal esperado. Já as variáveis capital humano e infraestrutura se apresentaram como importantes para o crescimento econômico. A estimativa para economias de aglomeração (população) apresentou sinal negativo, indicando que não há economias de aglomeração dessa fonte. O investimento doméstico não se apresentou como estatisticamente significante.

$\mathrm{Na}$ literatura brasileira existe uma lacuna sobre o entendimento da relação entre IED e crescimento econômico, principalmente considerando a questão espacial. Assim, o presente trabalho pode contribuir para a discussão acerca desse tema no Brasil sob dois enfoques: primeiro, com a aplicação do procedimento de dados em painel, em que se constatou que existe uma relação positiva entre IED e crescimento econômico; segundo, fazendo uso da análise espacial, cujos resultados apontaram para a existência de correlação espacial e externalidade positiva do IED entre os estados. Por fim, em termos de políticas a serem implementadas, os resultados desta pesquisa sugerem que a participação do IED nos estados não deve ser negligenciada.

\section{Referências}

ADKINS, L. C.; HILL, R. C. Using Stata for principles of econometrics. 4. ed. John Wiley $\mathcal{E}$ Sons, 2011.

AGOSIN, M. R.; MAYER, R. Foreign investment in developing countries: does It crowd in domestic investment? Geneva, Switzerland: UNCTAD, 2000. (UNCTAD Working paper n. 146).

ALFARO, L. et al. FDI and economic growth: the role of local financial markets. Journal of International Economics, v. 64, n. 1, p. 89-112, 2004.

ALFARO, L.; KALEMLI-OZCAN, S.; SAYEK, S. FDI, Productivity and financial development. The Word Economy, v. 32, n. 1, p. 111-135, 2009.

ALIBER, R. A theory of foreign direct investment. In: KINDLEBERGER, C. P. The international corporation. Cambridge: MIT Press, 1970.

ALMEIDA, E. S. Econometria espacial aplicada. Campinas: Alínea, 2012.

ANSELIN, L. Spatial econometrics. Dallas: Bruton Center, School of Social Sciences, University of Texas, 1999.

BANCO CENTRAL DO BRASIL. Censo de capitais estrangeiros no país. Disponível em: $<$ http://www.bcb.gov.br/?censoce > . Acesso em: 16 out. 2012.

BENGOA, M.; SANCHEZ-ROBLES, B. Foreign direct investment, economic freedom and growth: new evidence from Latin America. European Journal of Political Economy, vo. 19, n.3, p. 529-545, 2003. 
BLANCO, L. R. The spatial interdependence of FDI in Latin America. World Development, v. 40, n. 7, p. 1337-1351, 2012.

BLONIGEN, B. A. et al. FDI in space: spatial autoregressive relationships in foreign direct investment. European Economic Review, v. 51, n. 5, p. 1303-1325, 2007.

BODE, E., NUNNENKAMP, P. E WALDKIRCH, A. Spatial effects of foreign direct investment in US states. Kiel, Germany: Kiel Institute for the World Economy, July 2009. (Kiel Working Paper, n. 1535).

. Spatial effects of foreign direct investment in US states. Canadian Journal of Economics, v. 45, n. 1, p. 16-39, 2012.

BORTOLUZZO, M. M.; SAKURAI, S. N.; BORTOLUZZO, A. B. Allocation of foreign direct investment across Brazilian states. Estudos Econômicos, v. 43, n. 2, p. 241-269, 2013.

CAMERON, C. A.; TRIVEDI, P. K. Microeconometrics Using Stata. Stata Press, 2010.

CAPUCHO, T. O.; PARRÉ, J. L. Produção leiteira no Paraná: um estudo considerando os efeitos espaciais. In: ENCONTRO NACIONAL DA ASSOCIAÇÃO BRASILEIRA DE ESTUDOS REGIONIAS E URBANOS, 8., 2010, Juiz de Fora. Anais... Juiz de Fora: Aber, 2010.

CARKOVIC, M.; LEVINE, R. Does foreign direct investment accelerate economic growth? In: MORAN, T. H.; GRAHAM, E. M.; BLOMSTROM, M. (Ed.). Does foreign direct investment promote development? New methods, outcomes and policy approaches. Washington, DC: Institute for International Economics, 2005.

CARVALHO, A. X. Y.; ALBUQUERQUE, P. H. M. Tópicos em econometria espacial para dados cross-section. Brasília, DF: Ipea, 2010. (Texto para discussão, n. 1508).

CHUDNOVSKY, D.; LÓPEZ, A. Inversión extranjera directa y desarrollo: la experiencia del Mercosur. Revista de La CEPAL, n. 92, p. 7-23, ago. 2007.

CRESPO, N; FONTOURA, M. P. Determinants Factor of FDI Spillovers: what do we really know? World Development, v. 35, n. 3, 410-425, 2007.

DUNNING, J.; STOPFORD, J. Multinational enterprises: global trends and company performance. Basingstoke: Macmillan, 1983.

DURHAM, J. B. Absorptive capacity and the effects of foreign direct investment and equity foreign portfolio investment on economic growth. European Economic Review, v. 48, n. 2, p. 285-306, 2004.

HAUSMAN, J. Specification tests in econometrics. Econometrica, v. 46, n. 6, p. 1251-1271, 1978.

HYMER, S. H. The international operations of national firms, a study of direct foreign investment. Massachusetts: The MIT Press, 1960.

INSTITUTO DE PESQUISA ECONÔMICA E APLICADA (IPEA). Ipeadata. 2010. Disponível em: <www.ipeadata.gov.br>. Acesso em: 10 mar. 2011. 
JAVORCIK, B. S. Does foreign direct investment increase the productivity of domestic firms? Is search of spillovers through backward linkages. American Economic Review, v. 94, n. 3, 605-627, 2004.

JORDAAN, J. A.; RODRÍGUEZ OREGGIA, E. Regional growth in Mexico under trade liberalization: how important are agglomeration and FDI? Annals of Regional Science, v. 48, p. 179-202, 2012.

KINOSHITA, Y.; CAMPOS, N. Why does FDI go where it goes? New evidence from the transition economies. Ann Arbor: University of Michigan, The William Davidson Institute, 2003.

KRUGMAN, P. Increasing returns and economic geography. Journal of Political Economy, v. 99, n. 3, p. 483-99, 1991.

KRUGMAN, P. What's new about the New Economic Geography. Oxford Review of Economics Policy, v. 14, n. 2, p. 7-17, 1998.

MADARIAGA, N.; PONCET, S. FDI in Chinese cities: spillovers and impact on growth. The World Economy, v. 30, n. 5, p. 837-862, 2007.

PENROSE, E.T. Foreign Investment and the Growth of the Firm. The Economic Journal, v. 66, n. 262, p. 220-235, 1956.

PINTO, P. B. O.; ALMEIDA, E. S. Efeitos da abertura comercial na distribuição de renda: uma abordagem espacial para municípios brasileiros. In: ENCONTRO DE ECONOMIA DA REGIÃO SUL - ANPEC-SUL, 15., 2012, Porto Alegre. Anais... Porto Alegre: PUCRS, 2012.

SILVA, R. R.; BACHA, C. J. Acessibilidade e aglomerações na Região Norte do Brasil sob o enfoque da Nova Geografia Econômica. Nova Economia, v. 24, n. 1, p. 169-190, jan./abr. 2014.

SJÖHOLM, F. Productivity growth in Indonesia: the role of regional characteristics and direct foreign investment. Economic Development and Cultural Change, v. 47, n. 3, p. 559-584, 1999.

UNITED NATIONS CONFERENCE ON TRADE AND DEVELOPMENT. World Investment Report. 2010. Disponível em: <www.unctad.org/wir>. Acesso em: 10 mar. 2011.

Recebido em: 19/03/2014. Aceito em: 13/11/2015. 